\title{
Understanding medical students' empathy based on Enneagram personality types
}

\author{
HyeRin Roh', Kyung Hye Park², Hyo Jeong Ko ${ }^{3}$, Dong Kyu Kim³, Han Bin Son ${ }^{3}$, Dong Hyeok Shin ${ }^{3}$, \\ Seung Hyeon Lee, ${ }^{3}$ Hee Young $\mathrm{Jung}^{3}$ and Dong $\mathrm{Heo}^{3}$ \\ ${ }^{1}$ Department of Medical Education and the Institute for Medical Humanities, Inje University College of Medicine, \\ Busan, ${ }^{2}$ Department of Medical Education, Yonsei University Wonju College of Medicine, Wonju, and ${ }^{3}$ Department \\ of Medicine, Inje University College of Medicine, Busan, Korea
}

Purpose: High self-awareness can promote communication and empathy. The Enneagram is a well-known personality tool to enhance self-awareness. We evaluated differences in empathy among medical students using the Enneagram typology.

Methods: This cross-sectional study included first and second grade students at the Inje University College of Medicine. The Jefferson Scale of Empathy was used to measure empathy and the Korean Enneagram Personality Type Indicator was used for examining personality characteristics. Empathy scores were analyzed according to the Triads, Hornevian group, Harmonic group, and each Enneagram type.

Results: The Instinctive triad, the Withdrawns, and the Positive outlook group were the most common, and the Feeling triad, the Assertives, and the Emotional realness group were the least common. Students in the Feeling triad and the Dutifuls had higher compassionate care (CC) scores as compared to their counterparts. Type 2 and 6 students showed the two highest empathy and CC scores. The empathy score of type 3 students was the lowest. Type 7 had the lowest CC score but the highest perspective taking score.

Conclusion: These differences in empathy according to Enneagram personality types can be applied to medical education to maintain and improve medical students' empathy.

Key Words: Medical students, Personality, Empathy, Medical education

\section{Introduction}

Physician empathy is defined as the "ability to understand the patient's inner experiences and perspective and a capability to communicate this understanding [1]." It is essential for a good patient-physician relationship and successful clinical outcomes [2]. However, low levels of physician empathy and decline in medical education have been reported [3,4]. To encourage empathy in medical students and physicians, medical educators have emphasized on patients' illness experiences, perspectives, concerns, and expectations during clinical communication skills training [5]. However, this teaching and learning strategy targeting skills only may not be enough to improve empathy [6]. It has been reported that the continuous enhancement of the self-understanding of personal traits and promoting self-growth can also
Received: December 13, 2018 • Revised: February 7, 2019 • Accepted: February 8, 2019 Corresponding Author: Kyung Hye Park (https://orcid.org/0000-0002-5901-6088)

Department of Medical Education, Yonsei University Wonju College of Medicine, 20 Ilsan-ro, Wonju 26426, Korea

Tel: +82.33.741.0242 Fax: +82.33.742.5034 email: erdoc74@gmail.com
Korean J Med Educ 2019 Mar; 31(1): 73-82. https://doi.org/10.3946/kjme.2019.120 eISSN: 2005-7288

(C) The Korean Society of Medical Education. All rights reserved. This is an open-access article distributed under the terms of the Creative Commons Attribution Non-Commercial License (http:// creativecommons.org/licenses/by-nc/3.0/), which permits unrestricted non-commercial use, distribution, and reproduction in any medium, provided the original work is properly cited. 
encourage communication with patients [7].

Personality is believed to be composed of innate traits that determine the patterns of thoughts, feelings, and behaviours that influence interpersonal interaction [8]. It is reported that personality is significantly associated with the empathy of medical students and that it should be taken into account while developing programs to enhance empathy [9]. To assess personality traits associated with empathy, previous researchers have used the Five-Factor Model (FFM) [10,11], ZuckermanKuhlman Personality Questionnaire (ZKPQ) [12], Temperament and Character Inventory (TCI) [13], and Kupfer Detre Scale (KDS-3A) [14]. The FFM and ZKPQ explain core personality traits, but they do not define personality type $[15,16]$. Further, the TCI and KDS-3A focus more on treating pathological personality types and mood disorders $[14,17]$. To use medical students' personality types for empathy education, tools designed for the normal population, rather than a clinical population, can be more helpful because not all medical students may exhibit pathological characteristics. In addition, it would be desirable to explain personality traits as well as to identify the deep roots of drives and inner conflicts of human nature.

Enneagram is one of the well-known personality typologies. It is also known as one of the greatest tools to enhance self-awareness [18]. The goal of the Enneagram is to help people understand the mechanism involved in their personality, to help them free themselves from the limitations of their personality [19]. Therefore, the Enneagram not only describes each personality characteristic, but it also provides insights about the habitual systems of behaviours, emotions, and ideas of each personality. In addition, the Enneagram facilitates self-transcendence by attaining the state of presence [20-22]. The Enneagram considers personality as an artificial construct and a collection of internal defences and reactions related to the self and the world [21]. In the journey of self-discovery using the Enneagram, people can become aware of essential qualities in themselves. Thus it goes beyond the narrow concerns of the personality and helps people become more aware of the present moment [21]. Every person has one dominant personality type among the nine types. However, their personality contains all nine types [22].

The nine personality types of the Enneagram can be categorized into different groups of three, called Triads, the Hornevian groups, and the Harmonic groups. The Triads show the main different issues of our ego and blocked or distorted functions [22]. The Hornevian groups indicate the social style and how each type tries to have its needs met [22]. The Harmonic groups indicate coping style and how each type copes when they do not get what they want [22]. The present authors thought that grouping students based on Triads, Hornevian groups, or Harmonic groups can help them understand their communication style and patterns more easily. Furthermore, we thought that this grouping and personality typing using the Enneagram can help students identify the obstacles that limit their expression of empathy, and accordingly, they can let go of such obstacles.

There is growing use of the Enneagram for selfgrowth and development of relationships in the area of business, parenting, education, and other various fields [23-25]. However, it has rarely been used in studies on empathy in medical education. Enneagram helps people face, overcome and empathize with inner conflict. Overcoming and empathizing with their own inner conflict lead genuine empathy to others. Therefore, we assessed medical students' empathy levels and Enneagram types and then compared their empathy scores according to Triads, the Hornevian group, the Harmonic group, and each Enneagram type. Based on these findings, we 
suggested educational strategies for promoting empathy in medical students.

\section{Methods}

\section{Participants}

Questionnaires were distributed to 202 first and second grade, preclinical phase students at the Inje University College of Medicine. We chose students from the preclinical years because their empathy is expected in a good state of preservation and not biased by clinical clerkship experiences. The cross-sectional study was performed in the second semester at the end of 2016. The questionnaire comprised an empathy scale, an Enneagram type indicator, and demographic details of the participants. This study was previously approved by the Institutional Review Board for Human Research at Inje University Busan Paik Hospital (IRB approval no., 16-0200).

\section{The students' version of the Jefferson Scale of Empathy}

The students' version of the Jefferson Scale of Empathy (JSE-S) was used to evaluate the empathy levels of the participants. The JSE was developed to evaluate physicians' empathy and it is also available in medical students [26]. The JSE-S was translated into Korean in 2010 and was reported to have a good reliability (Cronbach's $\alpha$ of 0.84) [27].

The JSE-S has three underlying constructs of empathy, comprising 10 items on perspective taking (PT), eight on compassionate care $(\mathrm{CC})$, and two on standing in the patient's shoes (SP) [26]. Each item is rated on a 7-point Likert scale. The total score is the sum of all item scores, and a higher score indicates a higher degree of empathy (min=20, $\max =140$ ). The PT score, CC score, and SP score is the sum of the relevant item scores.

\section{Korean Enneagram Personality Type In- dicator}

The Korean Enneagram Personality Type Indicator (KEPTI) was used to assess the personality typology in this study. It is a standardized indicator that consists of 81 questions that are rated on a 5-point Likert scale (Cronbach's $\alpha=0.90$, retest reliability $=0.89$, construct validity with the Riso-Hudson Enneagram Personality Type Indicator=0.82) [28].

The Triads refers to the basic three components of the human psyche: instincts, feelings, and thinking (Table 1) [22]. Everyone person integrates his/her instincts, feelings, and ability to think when he/she is present, but each person is driven to be disintegrated when he/she is not present and develops imbalance in one of the triads. The Instinctive triad seeks autonomy when individuals are not present. They resist and want to control the environment. They tend to experience rage when they sense that they cannot control others or themselves. In addition, they experience anger when they sense others try to control them. It is also called as the body centre because the triad is concerned with body intelligence.

The Feeling triad is concerned with emotional intelligence and it seeks attention from others when they are not present [22]. These individuals create a false self-image and feel ashamed when they feel that the others are indifferent to their assumed quality of identity.

The Thinking triad seeks steadiness and such individuals try to find inner guidance and support when they are not present [22]. The Thinking triad pertains to cognitive intelligence. They tend to be anxious when they think something is unsafe. They try to create a sense of safety by thinking about strategy, consultation, and planning. The behaviours of each group to achieve 
Table 1. Interpretation of Groups of Triads, Hornevian Groups, and Harmonic Groups

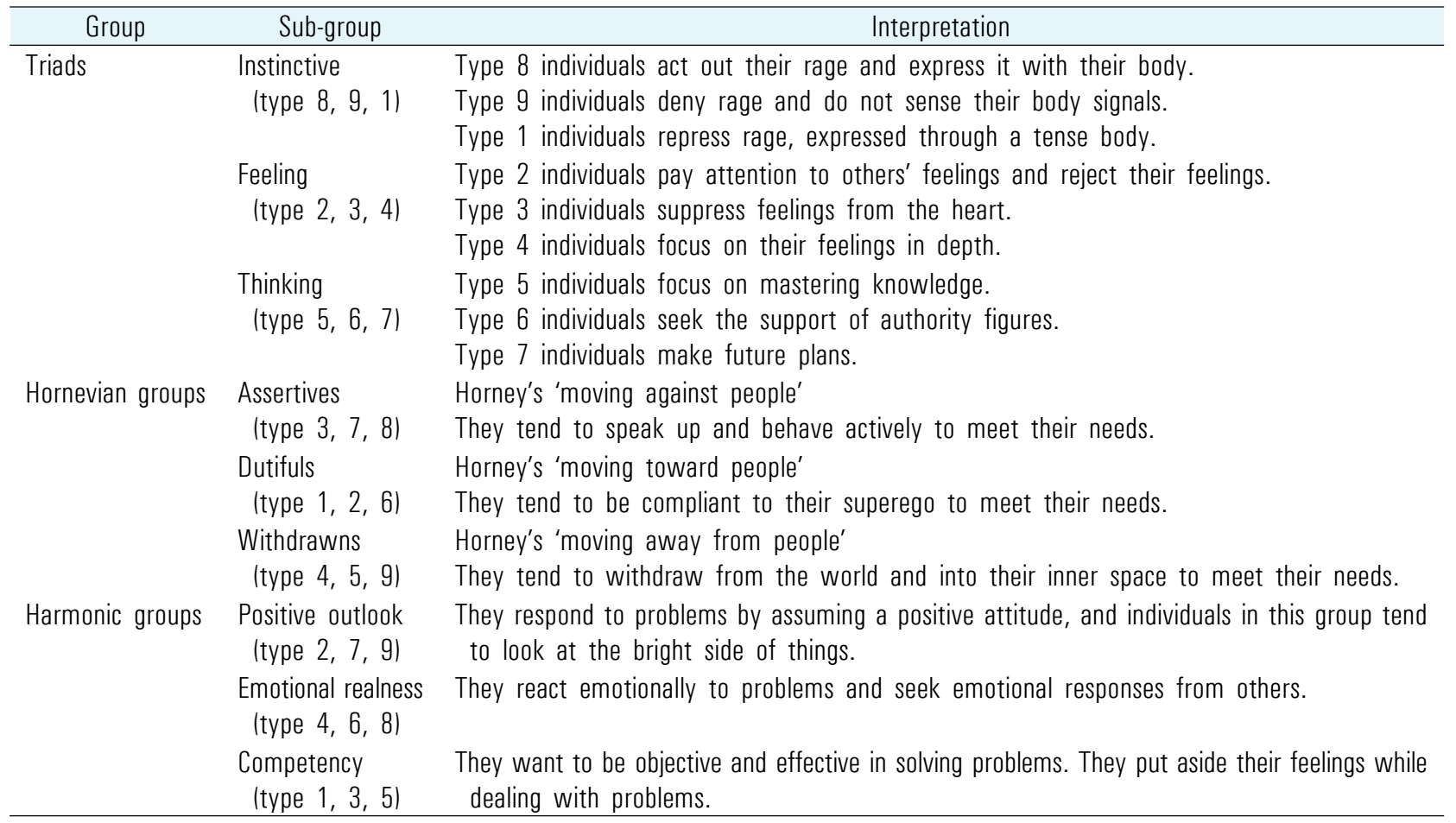

autonomy, false self-image, or steadiness prevent people from feeling and expressing empathy toward others.

The 'Hornevian groups' is a three-group categorization of the Enneagram personality types according to social styles when people are not present (Table 1) [29]. Riso and Hudson [22] named them the Hornevian groups in honour of Karen Horney, who reported that people tend to unconsciously assume one interpersonal type to maintain social security [30]. The 'Harmonic groups' is another three-group classification of the Enneagram types according to coping style when they are not present (Table 1) [22,29].

\section{Statistical analyses}

The Enneagram type of each respondent was defined based on the type that exhibited the highest score. Respondent's Triads, Hornevian group, and Harmonic group were defined using his/her Enneagram type. The JSE-S scores were compared according to the Triads, Hornevian group, and Harmonic group, using the
Kruskal-Wallis test with Bonferroni correction (posthoc analysis) for multiple comparisons; a probability value of $<0.017$ was considered significant. The PT, CC, and SP sub-categories of the JSE-S were also compared the same way. All continuous variables were then described as median values and interquartile ranges. The IBM SPSS ver. 23.0 (IBM Corp., Armonk, USA) was used for the analyses, and significance was declared at $\mathrm{p}<0.05$.

\section{Results}

\section{Demographics of the respondents and empathy score}

A total of 162 questionnaires were returned among 202, of which 16 questionnaires were excluded because of incomplete replies to the KEPTI or the Korean JSE-S. Since the ultimate objective of our study was not analysis 
according to demographic data, the questionnaires with incomplete demographic data were included. Consequently, 148 questionnaires were finally included.

The mean age of the respondents was $23.18 \pm 1.37$ years $(\mathrm{N}=130)$. Of the respondents, 81 were male and 49 were female, while 18 did not report their gender. Further, 38 of them were in the first grade of medical school, 46 were in the second grade, and 64 did not respond to this

Table 2. Demographic Characteristics of the Respondents and Empathy Scores

\begin{tabular}{lc}
\hline \multicolumn{1}{c}{ Variable } & Value \\
\hline Age (yr, $N=130)$ & $23.18 \pm 1.37$ \\
Sex $(\mathrm{N}=130)$ & $81(62.3)$ \\
Male & $49(37.7)$ \\
Female & \\
Grade ( $N=84)$ & $38(45.2)$ \\
First year & $46(54.8)$ \\
Second year & \\
Jefferson Scale of Empathy & $109(100.0-115.8)$ \\
Total & $56(52-60)$ \\
Perspective taking & $45(41-48)$ \\
Compassionate care & $7(6-10)$ \\
Standing in patient's shoes & \\
The Enneagram profiles of respondents & $27(18.2)$ \\
Type 1: the reformer & $15(10.1)$ \\
Type 2: the helper & $4(2.7)$ \\
Type 3: the achiever & $10(6.8)$ \\
Type 4: the individualist & $20(13.5)$ \\
Type 5: the investigator & $8(5.4)$ \\
Type 6: the loyalist & $7(4.7)$ \\
Type 7: the enthusiast & $4(2.7)$ \\
Type 8: the challenger & $53(35.8)$ \\
Type 9: the peacemaker &
\end{tabular}

Data are presented as mean \pm standard deviation, number $(\%)$, or median (interquartile range). item (Table 2). The median value on the Korean JSE-S was 109.0 (Cronbach's $\alpha=0.835$ ), while that on the PT, CC, and SP sub-domain was 56.0, 45.0, and 7.0, respectively (Table 2). The Cronbach's $\alpha$ of the Enneagram was 0.656 in the present study. Among all respondents, the type 9 personality was the most common, followed by type 1 and type 5 . Type 3 and 8 were the least common (Table 2).

\section{Empathy scores according to the Triads, Hornevian groups, and Harmonic groups}

Among the three Triads, the Instinctive triad was the most common. There was no difference in the total empathy score across the Triads. However, the CC score of the Feeling triad was significantly higher than that of the Instinctive triad was $(\mathrm{p}=0.014$ for the post-hoc analysis) (Table 3).

The Withdrawns were the most common among the Hornevian groups. Regarding the Hornevian groups, the median total empathy score exhibited a statistical difference, but the post-hoc analysis revealed no significant differences. The median value of the $\mathrm{CC}$ score of the Dutifuls was 48.0, which was significantly higher than that of the others (Table 4). The Positive outlook group was the most common, and there were no differences among the Harmonic groups (Table 5).

Table 3. Empathy Score according to the Enneagram Triads

\begin{tabular}{lcccc}
\hline \multirow{2}{*}{ Jefferson Scale of Empathy } & \multicolumn{3}{c}{ Triads } \\
\cline { 2 - 5 } & $\begin{array}{c}\text { Instinctive (type 8, 9, 1) } \\
(\mathrm{N}=83)\end{array}$ & $\begin{array}{c}\text { Feeling (type 2, 3, 4) } \\
(\mathrm{N}=27)\end{array}$ & $\begin{array}{c}\text { Thinking (type 5, 6, 7) } \\
(\mathrm{N}=38)\end{array}$ \\
\hline Total & $109.0(100.0-115.0)$ & $109.0(104.0-119.0)$ & $108.0(95.8-115.0)$ & 0.472 \\
Perspective taking & $55.0(51.0-61.0)$ & $58.0(53.0-60.0)$ & $58.0(52.5-61.0)$ & 0.799 \\
Compassionate care & $45.0^{\mathrm{a})}(42.0-48.0)$ & $47.0(43.0-51.0)$ & $44.0(39.0-47.3)$ & 0.031 \\
Standing in patient's shoes & $8.0(6.0-10.0)$ & $7.0(6.0-11.0)$ & $7.0(6.0-9.3)$ & 0.935 \\
\hline
\end{tabular}

Data are presented as median (interquartile range).

${ }^{a} p=0.014$ when compared with the Feeling triad. 
Table 4. Empathy Score according to the Enneagram Hornevian Group

\begin{tabular}{lcccc}
\hline \multirow{2}{*}{ Jefferson Scale of Empathy } & \multicolumn{4}{c}{ Group } \\
\cline { 2 - 5 } & $\begin{array}{c}\text { Assertives (type 3, 7, 8) } \\
(\mathrm{N}=14)\end{array}$ & $\begin{array}{c}\text { Dutifuls (type 1, 2, 6) } \\
(\mathrm{N}=53)\end{array}$ & $\begin{array}{c}\text { Withdrawns (type 4, 5, 9) } \\
(\mathrm{N}=81)\end{array}$ & $\mathrm{p}$-value \\
\hline Total & $106.0(88.5-111.5)$ & $111.0(104.0-121.0)$ & $107.0(99.0-114.5)$ & 0.030 \\
Perspective taking & $57.5(47.8-62.3)$ & $57.0(52.5-61.5)$ & $56.0(51.0-59.0)$ & 0.512 \\
Compassionate care & $41.5(34.3-46.3)$ & $48.0^{\mathrm{a}), \mathrm{b})}(44.0-50.0)$ & $44.0(40.0-47.0)$ & 0.001 \\
Standing in patient's shoes & $7.0(6.0-8.0)$ & $8.0(6.0-10.0)$ & $7.0(6.0-9.5)$ & 0.250 \\
\hline
\end{tabular}

Data are presented as median (interquartile range).

${ }^{\text {a) }}=0.014$ when compared with the Assertives. ${ }^{b /} p<0.001$ when compared with the Withdrawns.

\begin{tabular}{lcccc}
\hline Table 5. Empathy Score according to the Enneagram Harmonic Group & \\
\hline \multirow{3}{*}{ Jefferson Scale of Empathy } & \multicolumn{4}{c}{ Group } \\
\cline { 2 - 4 } & $\begin{array}{c}\text { Positive outlook group } \\
\text { (type 2, 7, 9) (N=74) }\end{array}$ & $\begin{array}{c}\text { Emotional realness group } \\
\text { (type 4, 6, 8) (N=24) }\end{array}$ & $\begin{array}{c}\text { Competency group } \\
\text { (type 1, 3, 5) (N=50) }\end{array}$ & \\
\hline Total & $107.0(99.8-115.3)$ & $108.5(102.8-116.3)$ & $110.5(99.8-116.8)$ & 0.529 \\
Perspective taking & $55.0(51.0-59.3)$ & $59.0(53.0-61.8)$ & $56.0(52.0-61.3)$ & 0.439 \\
Compassionate care & $44.0(41.0-48.0)$ & $46.0(40.3-49.8)$ & $45.0(41.0-48.0)$ & 0.672 \\
Standing in patient's shoes & $7.0(6.0-9.3)$ & $6.5(6.0-9.8)$ & $8.0(6.8-10.0)$ & 0.086 \\
\hline
\end{tabular}

Data are presented as median (interquartile range).

\section{Fig. 1. Median Values of the Total and Sub-Domain Empathy Score according to Enneagram Types}

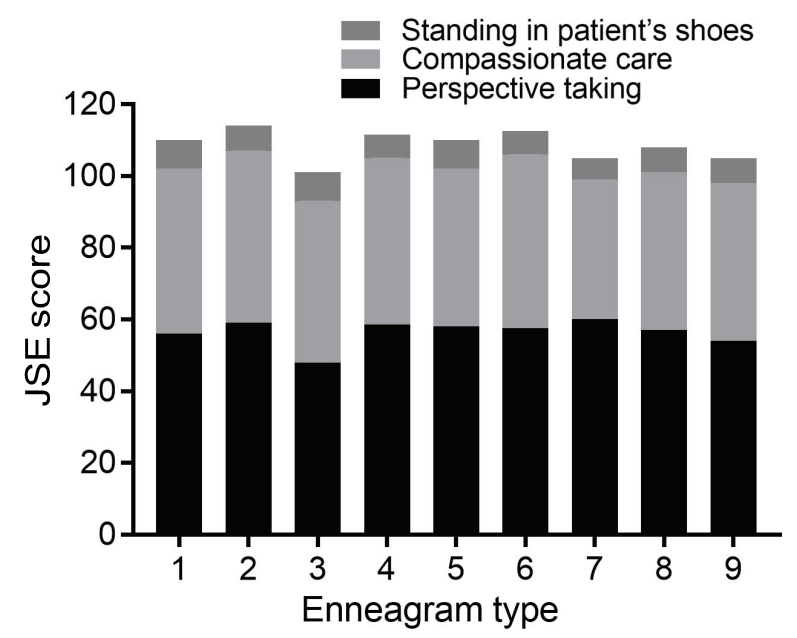

\section{Empathy scores according to Enneagram types}

The JSE-S score for the type 2 personality was 117.0, which was the highest empathy score. This type was followed by type 6. Type 3 scored the lowest (Fig. 1). The CC score for type 6 was 48.5, which was the highest among all the types. It was followed by type 2 . The CC score of type 7 was the lowest, but the PT score of this type was the highest among the nine types (Fig. 1).

\section{Discussion}

Empathy is important for medical students' communication and professionalism [18]. Therefore, there should be specific plans to maintain or improve empathy in medical students. This study examined scores on an empathy scale according to personality types using the Enneagram. The Enneagram typology is not commonly used in the medical education field; therefore, this study could draw new insights about the use of this typology for designing medical education for empathy based on students' personality types.

In this study, the Instinctive triad, the Withdrawns, and the Positive outlook groups were the most commonly observed personality categories, and the Feeling triad, the Assertives, and the Emotional realness groups were the least common. Further, among individual per- 
sonality types, type 9,1 , and 5 were more common, while type 3 and 8 were the least common. These findings are similar to those of studies of medical students and other populations in Korea, which revealed that type 9 and 1 were the two most common types and type 3 was the least common one [18,31-33]. However, these studies identified different types as the third most common, including type 2, 5, and 7 [18,31,32]. The distribution of types could be uneven owing to nationality or the culture of the organization [25]. Thus, the present results may indicate that the culture of Korea and that of medical schools may have led to the dominance of type 9 and 1 personalities, the essential qualities of which are harmony and integrity. Type 9 and 1 individuals fear being emotionally aggressive when they are not present. On the other hand, the least common personalities, i.e., type 3 and 8 , are characterized by assertiveness. The common appearance of type 5 can be explained by the fact that this type tends to focus on knowledge and cognition, which are emphasized in medical schools.

Students in the Feeling triad had the highest CC score. The strength of the Feeling triad lies in being able to express and understand feelings in depth, and it is related to emotional intelligence. $\mathrm{CC}$ is related to the recognition and understanding of patients' emotions [26]. Thus, these aspects can explain the higher CC scores of those in the Feeling triad. The Feeling triad exhibits better immediacy in facilitative communication, and such individuals tend to use more noticing and companioning in interpersonal caring behaviour [34,35]. Emotional intelligence has a key influence on the patient -physician interaction [36]. Therefore, in terms of communication education, encouraging students to develop feeling-centred characteristics may be helpful for enhancing the $\mathrm{CC}$ domain of empathy.

Among the Hornevian groups, the Dutifuls had the highest CC score in the present study. The Dutifuls tend to obey principles and rules and they easily adapt their responsibilities and liabilities to the rules [22]. They strongly feel the need to help others and try to identify what is right to do. They tend to suppress their own wishes and strictly push themselves to be responsible for other people, and for the organization and the society. Therefore, when they encounter patients, we can expect that such individuals will try to do their duty as a doctor, as best as they can. In terms of communication education, encouraging students to develop qualities of the Dutifuls may be helpful to enhance the CC domain of empathy. However, the Dutifuls have problems with applying principles too strictly and rigidly, and they tend to treat themselves harshly. Therefore, it is important to educate the Dutifuls to strike a balance between empathy toward others and empathy toward the self.

Among personality types, type 2 had the highest empathy scores in this study. This finding can be explained by type 2's essence. According to the Enneagram theory, type 2's essential quality is unconditional love [19]. These individuals search for intimate relationships, and they are caring and loving. They are empathetic and compassionate when they are present [19]. Therefore, encouraging students to develop these qualities may help enhance their empathy.

Type 6 had the highest CC score in the present study. This finding can be explained by the type 6's basic fear and desire. According to the Enneagram theory, the basic fear of type 6 individuals is not having support and guidance [22]. They truly understand how anxious people can feel when there is no support. Therefore, they try to be loyal to friends and support others when they are present. In terms of empathy, type 6 individuals $a b^{-}$ solutely understand patients' anxiety and try to support and guide them. These behaviours can cause the patients feel safe and to consider their doctor as empathetic. 
Encouraging students to develop type 6 qualities could enhance their empathy.

Type 3 had the lowest empathy score in the present study. Type 3 is one of the types in the Feeling triad. Thus, these individuals are good at perceiving emotions. However, they tend to ignore their own emotions when they are present [22]. Instead, they try to be efficient and to succeed in their job [21]. These characteristics may explain their low empathy scores. Thus, when enhancing the empathy of type 3 students, deep reflection on, and recognition of their tendency to ignore their feelings would be helpful.

The present study revealed an interesting result regarding type 7 students, who had the lowest CC score but the highest PT score. According to the Enneagram theory, the basic fear of type 7 individuals is that of deprivation and being trapped in emotional pain. They always want to be happy and they love having fun [21]. They are afraid of sustaining emotional suffering. They seek happier experiences when they encounter sufferings and boredom. These aspects could partly explain their low CC score. On the other hand, they had the highest PT score. PT is the core cognitive component of empathy and the inclination to adopt the beliefs and feelings of patients [26]. Type 7 is one of the types in the Thinking triad, which focuses on thinking, which could partly explain the high PT score. Therefore, we can expect that type 7 individuals understand patients' emotions by thinking and not by feeling. Thus, when enhancing the empathy of type 7 individuals, deep reflection on their basic fear, and recognition of their tendency to think rather than to feel, would be helpful.

There are a few limitations in this study. This study was conducted in a single medical college and it recruited only preclinical medical students. Additionally, as the present study was restricted to Korea, it may have been influenced by specific dominant types. The Enneagram profile in other countries may be different from those observed in the present study, owing to cultural factors. Therefore, cultural considerations are necessary while interpreting the results of the Enneagram. Second, although KEPTI has good validity and reliability, Enneagram test is still a controversial theory in psychological field. In addition, since classification of nine personality types is based on determinism and may be not scientific, utilizing results should be cautious.

In conclusion, we found some differences in empathy according to Enneagram types. We suggest that personality traits should be taken into consideration while designing programs to enhance empathy through medical education. It is necessary to teach medical students to deal with emotions, especially in the CC domain, while fulfilling doctors' other responsibilities. Medical students with low empathy can be encouraged to develop the personality traits that are potentially related to good empathy. Enneagram type can be used for medical students to understand their own strength and weakness of empathic ability. Their weakness can be overcome through reflection, and empathy can be maintained and improved. Our results would be useful for medical communication teachers as they can teach empathy to medical students based on their personality characteristics.

\section{ORCID:}

HyeRin Roh: https://orcid.org/0000-0002-3879-709X; Kyung Hye Park: https://orcid.org/0000-0002-5901-6088; Hyo Jeong Ko: https://orcid.org/0000-0003-2777-798X; Dong Kyu Kim: https://orcid.org/0000-0002-0742-6325; Han Bin Son: https://orcid.org/0000-0001-6917-2239; Dong Hyeok Shin: https://orcid.org/0000-0002-6570-6958; Seung Hyeon Lee: https://orcid.org/0000-0002-1166-1918; Hee Young Jung: https://orcid.org/0000-0002-5534-6119; 
Dong Heo: https://orcid.org/0000-0002-4996-3225

Acknowledgements: None.

Funding: None.

Conflicts of interest: No potential conflict of interest relevant to this article was reported.

Author contributions: KHP: conception or design of the work and critical revision of the article; HJK, DKK, HBS, DHS: data collection; SHL, HYJ, DH: data analysis and interpretation; HR: drafting the article; and all authors: final approval of the version to be published.

\section{References}

1. Hojat M, Gonnella JS, Nasca TJ, Mangione S, Vergare M, Magee M. Physician empathy: definition, components, measurement, and relationship to gender and specialty. Am J Psychiatry. 2002;159(9):1563-1569.

2. Stepien KA, Baernstein A. Educating for empathy: a review. J Gen Intern Med. 2006;21(5):524-530.

3. Kelm Z, Womer J, Walter JK, Feudtner C. Interventions to cultivate physician empathy: a systematic review. BMC Med Educ. 2014;14:219.

4. Sng G, Tung J, Ping YS, et al. Complex and novel determinants of empathy change in medical students. Korean J Med Educ. 2016;28(1):67-78.

5. Silverman J, Kurtz S, Draper J. Skills for communicating with patients. 3rd ed. Boca Raton, USA: CRC Press; 2013.

6. Spencer J. Decline in empathy in medical education: how can we stop the rot? Med Educ. 2004;38(9):916-918.

7. Novack DH, Suchman AL, Clark W, Epstein RM, Najberg E, Kaplan C. Calibrating the physician: personal awareness and effective patient care. Working Group on Promoting Physician Personal Awareness, American Academy on Physician and Patient. JAMA. 1997;278(6): 502-509.

8. Atkinson RL, Atkinson RC, Smith EE, Bem DJ, Nolen-
Hoeksema S. Hilgard's introduction to psychology. Ankara, Turkey: Arkadaş Publishing; 1999.

9. Magalhães E, Costa P, Costa MJ. Empathy of medical students and personality: evidence from the Five-Factor Model. Med Teach. 2012;34(10):807-812.

10. Costa P, Alves R, Neto I, Marvão P, Portela M, Costa MJ. Associations between medical student empathy and personality: a multi-institutional study. PLoS One. 2014; 9(3):e89254.

11. Song Y, Shi M. Associations between empathy and big five personality traits among Chinese undergraduate medical students. PLoS One. 2017;12(2):e0171665.

12. Hojat M, Zuckerman M, Magee M, et al. Empathy in medical students as related to specialty interest, personality, and perceptions of mother and father. Pers Individ Differ. 2005;39(7):1205-1215.

13. Lee BK, Bahn GH, Lee WH, Park JH, Yoon TY, Baek SB. The relationship between empathy and medical education system, grades, and personality in medical college students and medical school students. Korean J Med Educ. 2009;21(2):117-124.

14. Kupfer DJ, Drew FL, Curtis EK, Rubinstein DN. Personality style and empathy in medical students. J Med Educ. 1978;53(6):507-509.

15. McCrae RR, John OP. An introduction to the five-factor model and its applications. J Pers. 1992;60(2):175-215.

16. Zuckerman M. Zukerman-Kuhlman Personality Questionnaire (ZKKPQ): an alternative five-factorial model. In: de Raad B, Perugini M, eds. Big Five Assessment. Seattle, USA: Hogrefe \& Huber Publishers; 2002:376-392.

17. Cloninger CR, Svrakic DM, Przybeck TR. A psychobiological model of temperament and character. Arch Gen Psychiatry. 1993;50(12):975-990.

18. Roh HR. Applying the enneagram to enhance self awareness in medical communication class. J Enneagram Stud. 2010;7(7):143-157.

19. Riso DR, Hudson R. Understanding the enneagram: the 
practical guide to personality types. Boston, USA: Houghton Mifflin Harcourt; 2000.

20. Riso DR. Enneagram transformations: releases and affirmations for healing your personality type. New York, USA: Houghton Mifflin Harcourt; 1993.

21. Riso DR, Hudson R. Personality types: using the Enneagram for self-discovery. New York, USA: Houghton Mifflin Harcourt; 1996.

22. Riso DR, Hudson R. The wisdom of the enneagram: the complete guide to psychological and spiritual growth for the nine personality types. 2nd ed. New York, USA: Bantam; 1999.

23. Bae KE, Park YK. Enneagram personality types analysis of learning styles in nursing students. J Enneagram Stud. 2014; $11(2): 94-114$.

24. Kingma M. Utilising a personality typology to resolve subliminal conflict in the workplace [dissertation]. Cape Town, South Africa: Cape Peninsula University of Technology; 2007.

25. Riso DR, Hudson R. Discovering your personality type: the essential introduction to the Enneagram: revised and expanded. New York, USA: Houghton Mifflin Harcourt; 2003.

26. Hojat M. Empathy in patient care: antecedents, development, measurement, and outcomes. New York, USA: Springer; 2007.

27. Roh MS, Hahm BJ, Lee DH, Suh DH. Evaluation of empathy among Korean medical students: a crosssectional study using the Korean version of the Jefferson Scale of Physician Empathy. Teach Learn Med. 2010;
22(3):167-171.

28. Youn YS. The enneagram personality type scale: its development and validation. J Educ Psychol. 2001;15(3): 131-161.

29. Hudson R. The Enneagram Harmonics workshop; Taipei City, Taiwan. Stone Ridge, USA: The Enneagram Institute; 2016.

30. Horney K. Our inner conflicts: a constructive theory of neurosis. New York, USA: Norton \& Company; 1992.

31. Choi MA. Differences in quality of life in accordance with the Enneagram personality types of medical students [master's thesis]. Gimhae, Korea: Inje University; 2016.

32. Hur Y, Lee KH. Analysis of medical students' enneagram personality types, stress, and developmental level. Korean J Med Educ. 201 1;23(3):175-184.

33. Youn YS. The analysis about the Korean measured by Korean Enneagram Personality Type Indicator. J Enneagram Stud. 2006;3(1):9-22.

34. Chung HJ. A study on self-expression and facilitative communication by Enneatype among university students. Korean J Community Living Sci. 2006;17(4):163-173.

35. Son YS. Interpersonal caring behavior and job satisfaction according to the Enneagram personality types of nurses medium sized hospital [master's thesis]. Cheonan, Korea: Dankook University; 2011.

36. Kim SH, Ko JK, Park JH. Effect of emotional intelligence on patient-physician interaction scores of clinical performance examination. Korean J Med Educ. 201 1;23(3): 159-165. 